\title{
Detection of Critical Situation of Longitudinal Vehicle Dynamics
}

\author{
Widya Nila Velayati ${ }^{1}$ and Sebastien Varrier $^{2}$
}

\begin{abstract}
This research presents a method of detecting critical situation for longitudinal vehicle dynamics. First, the dynamical system is modeled that consists of longitudinal tire force and longitudinal aerodynamic drag force. The longitudinal tire force is well known to be highly nonlinear. However, one can classify it in three types of operation : normal zone, critical zone and skidding zone. Normal zone represents the linear part of longitudinal tire force while in the critical zone, the vehicle does no longer correspond to the linear system. The difference between the nonlinear force and its linear model is considered as a fault that has to be detected. The aim of this project is to detect that fault. As the system depends on the speed of the vehicle, a Linear Parameter Varying (LPV) dynamical system is considered and an LPV fault detection approach is handled. An extension of the parity-space approach for LPV systems is applied to detect the fault on the vehicle. In order to accommodate the existence of the faults, the $\mathrm{H} \infty$ robust control system has been designed. Afterward, it is found that the controller can accommodate the faults. This research have been also implemented on a 1/5th scale vehicle by the simulation.
\end{abstract}

Keywords - LPV, Parity Space Approach, Hœ Robust Control System.

\section{INTRODUCTION}

$\mathrm{R}$ egarding the demand of automotive market in comfort and safety aspect that increase during the last 30 years, it is important to develop intelligent system in these aspects. Due to the fact that the development of process increases, it will be more complex and faults on systems can lead to serious consequences. In this part, the role of robust control /uncertain modeling is important since it permits to omit unmodeled dynamics as almost all physical systems are not perfectly known. Due to the issue of fault detection and isolation (FDI) strategies, different model-based methods have been used. Analytical redundancy-based methods are the most handled approaches including parity space approach implemented by [1].

The main work of this research is to apply fault detection approach which is presented in [1] and design Hळ robust control to the LPV system. In this work, the $1 / 5$ th scale vehicle is chosen as LPV system which is modeled by LTI system with dependant parameter. For this research, the following works should be done.

a. Modeling non linear system

b. Implementation fault detection approach

c. Design an Ho robust control to the LPV system

d. Analyzing the stability of the robust control system

In this paper, it is necessary to divide into three parts to introduce these works. The first part represents the LPV modeling of the $1 / 5$ th scale vehicle. In the second part, the methods which are used will be presented. It consists of parity space approach for fault detection and an $\mathrm{H} \infty$ robust synthesis to control to the LPV system. The result is presented in the third part. Finally, in the last part, the conclusion of the whole work is presented

\section{SYSTEM DESCRIPTION AND MODELING}

\section{A. Linear Parameter Varying (LPV)}

The first step of the LPV approach consists in "translating" (if necessary) the nonlinear model into a LPV one. The general idea consists in finding a

\footnotetext{
${ }^{1}$ Widya Nila Velayati is with Universite Joseph Fourier, Grenoble, France. E-mail: vela3391@yahoo.com.

${ }^{2}$ Sebastien Varrier is with Gipsa-Lab, Grenoble, France.
}

transformation that turns the nonlinear model into a linear parameterized one. This parameterized (LPV) model should match the whole nonlinear system state space range.

A nonlinear system can be described, in a non unique way, as a LPV system. The general aim is to find $\rho=\sigma(x) \in \mathrm{P}_{\rho}$ such that the LPV model is equivalent to the nonlinear one, i.e.:

$$
A(\sigma(x)) x+B(\sigma(x)) w=f(x, w)
$$

where $f(x, w)=\dot{x}$ is the nonlinear dynamical system equation and $\rho=\sigma(x)$ is known and depends on the measured signal. The main problem is obviously to find such $\sigma($.) function.

According to the previous nonlinear and LTI dynamical system definitions, a natural extension of the LTI definition lies in the LPV system description which gives somehow a trade-off between nonlinear and LTI formulations, as described thereafter.

Given the linear matrix functions $A \in \mathfrak{R}^{n x n}, B \in \mathfrak{R}^{n x n_{w}}, C \in \mathfrak{R}^{n_{z} x n}$ and $D \in \mathfrak{R}^{n_{z} x n_{w}}$, a Linear Parameter Varying (LPV) dynamical system $\left(\Sigma_{\mathrm{LPV}}\right)$ can be described as:

$$
\Sigma_{\mathrm{LPV}}:\left\{\begin{array}{l}
\dot{x}(t)=A(\rho(.)) x(t)+B(\rho(.)) w(t) \\
z(t)=C(\rho(.)) x(t)+D(\rho(.)) w(t)
\end{array}\right.
$$

where $x(t)$ is the state which takes values in a state space $X \in \mathfrak{R}^{n}, w(t)$ is the input taking values in the input space $W \in \mathfrak{R}^{n_{w}}$ and $z(t)$ is the output that belongs to the output space $Z \in \mathfrak{R}^{n_{s}}$. Then, $\rho=\sigma($.$) is a varying$ parameter vector that takes values in the parameter space $\mathrm{P}_{\rho}$ (a convex set) such that,

$$
\begin{gathered}
\mathrm{P}_{\rho}:=\left\{\rho(.):=\left[\begin{array}{lll}
\rho_{1}(.) & \ldots & \rho_{l}(.)
\end{array}\right]^{T} \in \mathfrak{R}^{l}\right. \text { and } \\
\left.\rho_{i} \in\left[\begin{array}{ll}
\underline{\rho_{i}} & \bar{\rho}_{i}
\end{array}\right] \forall_{i}=1, \ldots, l\right\}
\end{gathered}
$$

where $l$ is the number of varying parameters. Then, from a general viewpoint, 
a. $\quad \rho()=.\rho$ a constant value, $\Sigma_{\mathrm{LPV}}$ is a Linear Time Invariant (LTI) system.

b. $\quad \rho()=.\rho(t), \Sigma_{\mathrm{LPV}}$ is a Linear Time Varying (LTV) system, where the parameter is a priori known.

c. $\rho()=.\rho(x(t)), \Sigma_{\mathrm{LPV}}$ is a quasi-Linear Parameter Varying (qLPV) system.

d. $\quad \rho()=.\rho(t)$ and an external parameter, $\Sigma_{\mathrm{LPV}}$ is an LPV system.

An LPV system has a linear state space representation but the matrices are dependent on the varying parameters. Then, a LPV system can be viewed as a combination of LTI systems, or, in some specific cases as a Linear Differential Inclusion (LDI).

The advantage of such a representation, among others, is that it allows to model nonlinear parameters description, while keeping the linear structure. Then it allows the use of tools of the linear control theory (with some slight modifications). In other words, LPV systems can model nonlinear plants through the linearization of these nonlinear models along the trajectories of $\rho$. The difference between LTI (Linear Time Invariant) and LPV (Linear Parameter-Varying) lies on the scheduling parameters.

\section{B. Modeling of The System}

The vehicle dynamics are influenced by longitudinal tire forces, aerodynamic drag forces, and gravitational forces. Figure 1 represents longitudinal forces acting on a vehicle moving on an inclined road. A force balance along the vehicle longitudinal axis yields

$$
\begin{gathered}
m \dot{V}_{x}=F_{x}-F_{\text {aero }} \\
I \dot{\omega}=-R F_{x}+T_{i}
\end{gathered}
$$

where $m$ represents mass of the vehicle. $V x$ and $\omega$ is respectively a longitudinal speed and rotational speed of the vehicle. With $I$ being the wheel moment of inertia, $R$ being the tire radius in meter, and $T_{i}$ being the torque inwheel motor. $F_{x}$ is longitudinal tire forcé Moreover, $F_{\text {aero }}$ is the equivalent longitudinal aerodynamic drag force where the definition of $F_{\text {aero }}$ is

$$
F_{\text {aero }}=k_{\text {aero }} V_{x}
$$

where $k_{\text {aero }}$ is the aerodynamic drag coefficient. One of the most used in the community is the model of Pacejka or longitudinal force $F_{x}$ is given as a function of the angle of drift wheel,

$$
F_{x}=D \sin [C \arctan (B \lambda-E(B \lambda-\arctan (B \lambda)))]
$$

This model is based on experimental measurement that used to refine parameters $B, C, D, E$ and where $\lambda$ is longitudinal slip ratio. The graphic representation of this function is presented in Figure 2. From this model, one finds three operation areas(Figure 2):

a. normal : where the tire friction is proportional to the slip ratio $\lambda$. Note that remaining in this zone is nice for control purpose since it is kept advantage of the linear structure.

b. critical : this is the area where the tire friction is almost maximal. c. skidding zone : in this area, the tire friction decreases.

Thus, in normal operation, the expression of longitudinal tire force $F_{x}$ is

$$
\overline{F_{x}}=C_{i j} \lambda
$$

where the $C_{i j}$ is the slope of the linear part. In this work is considered the accumulative tire stiffness among the four tires. Longitudinal tire/road models, that defines the longitudinal friction force between the road and the tire contact path. Such a force is mainly characterized by the slip ratio $(\lambda)$ which defines the relative speed between the longitudinal speed of the wheel and the linear rotational speed of the wheel, defined as

$$
\lambda=\left\{\begin{array}{cc}
\frac{R \omega-V_{x}}{V_{x}}, & \text { during breaking } \\
\frac{R \omega-V_{x}}{R \omega}, & \text { during accelerating }
\end{array}\right.
$$

During accelerating, linear rotational speed $(R \omega)$ must be greater than the longitudinal speed $\left(V_{x}\right)$. While during breaking, linear rotational speed $(R \omega)$ must be smaller than the longitudinal speed $\left(V_{x}\right)$. In order to take nonlinear aspect of the tire, the longitudinal tire force contains linear and non-linear part. Then, it can be defined as

$$
F_{x}=\overline{F_{x}}+\Gamma
$$

We note that in the linear area (normal), the difference between the two models is small, while in the critical area, the difference is much more significant.

From the model given, longitudinal vehicle dynamics can be represented as

$$
\left[\begin{array}{c}
\dot{V}_{x} \\
\dot{\omega}
\end{array}\right]=\left[\begin{array}{cc}
-\frac{k_{\text {aero }}}{m}-\rho \frac{C_{i j}}{m} & \rho \frac{C_{i j} R}{m} \\
\rho \frac{C_{i j} R}{I} & \rho \frac{C_{i j} R^{2}}{I}
\end{array}\right]\left[\begin{array}{c}
V_{x} \\
\omega
\end{array}\right]+\left[\begin{array}{c}
0 \\
\frac{\alpha}{I}
\end{array}\right] u_{i}+\left[\begin{array}{c}
1 / m \\
1 / I
\end{array}\right] \Gamma
$$

The parameter variant $\rho=\frac{1}{V_{x}}$ for braking and $\rho=\frac{1}{R \omega}$ for accelerating. The problem of detection of the critical situation is a problem of faults detection where $\Gamma$ is faults which is detected. Therefore, the practical observation is done to obtain the parameter shown in Table 1.

\section{FAULT DETECTION AND RoBUST CONTROL METHODOLOGIES}

In this work, there are two problems. The first problem is the fault detection to detect the nonlinear tire force. This problem will be handheld by the parity space approach which is presented in the first section. Afterwards, the second problem is how to accommodate the fault or how to run in the normal zone. The $\mathrm{H}_{\infty}$ robust control is proposed to solve the problem. It will be presented in the second section.

\section{A. Parity Space Approach for Faults Detection}

Fault detection and isolation are important to improve the safety and reliability of practical control systems. The fault informators called residuals are small if the system is operating normally, but are large if there are faults in the system. This report evaluate the parity space approach in detecting faults in a discrete state space system. The goal is to detect faults that exists in LPV system. The system is represented by, 


$$
\begin{gathered}
x(k+1)=A x(k)+B u(k)+E f(k) \\
y(k)=C x(k)+D u(k)+F f(k)
\end{gathered}
$$

Applying parity-space approach, the output $y$ is expressed along a horizon s. It is obtained the following expression:

$$
Y_{s}(k)-G_{a b d} U_{S}(k)=H_{o s} x(k)+G_{A E F} F_{S}(k)
$$

where

$$
\begin{gathered}
Y_{S}=\left[\begin{array}{c}
y(k) \\
y(k+1) \\
\vdots \\
y(k+s)
\end{array}\right], U_{s}=\left[\begin{array}{c}
u(k) \\
u(k+1) \\
\vdots \\
u(k+s)
\end{array}\right] H_{o s}=\left[\begin{array}{c}
C_{0} \\
C_{0} A_{0} \\
\vdots \\
C_{0} A_{0}{ }^{s-1}
\end{array}\right], \\
G_{A B D}=\left[\begin{array}{ccccc}
D & 0 & \cdots & \cdots & 0 \\
C_{0} B & D & 0 & \cdots & 0 \\
C_{0} A_{0} B & C_{0} B & D & \ddots & \vdots \\
\vdots & \ddots & \ddots & \ddots & 0 \\
C_{0} A_{0}^{s-2} B & C_{0} A_{0}^{s-1} B & \cdots & C_{0} B & D
\end{array}\right],
\end{gathered}
$$

$x(k)$ and $F_{s}(k)$ have the similar formula with $Y_{s}(k)$. And we can make $G_{A E F}$ by seeing the formula of $G_{A B D}$.

a. Definition 1:

The parity equation at time $k$ is given by:

$$
r=W(\rho(k))^{T}\left(Y_{S}(k)-G_{A B D}(\rho(k)) U_{S}\right)
$$

where, $r(k)$ is the residual. $r(k)$ is decoupled from the system state when the system is normal and it is identically zero if the unknown non linear terms are identically zero. Otherwise, $r(k)$ is nonzero, it means that there are faults.

b. Definition 2:

Matrix $W$ is selected as a basis for the nullspace of $H_{o s}$ (orthogonal by $H_{o s}, W \perp H$ ). Therefore,

$$
W^{T} H_{o s}=0
$$

\section{B. $H_{\infty}$ Robust Control System}

The objectives of any control system [Skogestad and ostlethwaite(1996)] is to shape the response of the system to a given reference and get (or keep) a stable system in closed-loop, with desired performances, while minimising the effects of disturbances and measurement noises, and avoiding actuators saturation, this despite of modeling uncertainties, parameter changes or change of operating point.

In this paper, it is chosen a method of performance analysis using sensitivity function. Then, small gain theorem is applied to observe the stability of close loop system.

\section{METHOD}

Acquired data for this study are from an air reciprocating compressor, with the following specification:
a. Type
b. Speed
c. Power
d. Drive
: single stage, single acting
: $630 \mathrm{rpm}$
: 2 HP
: electrical motor 380 volt, $1430 \mathrm{rpm}$
e. Working pressure

f. Speed reduction : belt transmission

The two variables being measured are vibration acceleration of a cylinder head and a pressure of a cylinder chamber. Both variables are taken simultaneously, and then are plotted against a crank angle. Measurement at the same time is triggered by used of a tachometer. Measurement data are recorded by a data logger, which in turn are downloaded to a digital computer, so that cylinder chamber pressure and vibration graphs can be displayed. Varied working pressures are conducted to verify the effects of modified valve seat profiles.

On the outer side of a cylinder head is placed an accelerometer equipped with a magnetic base to measure the ensuing vibration. A hole is drilled in the cylinder head to place a pressure transducer, so that a chamber pressure can be measured. The vibration and the cylinder chamber pressure are measured at the same time, i.e. both signals are measured at the corresponding crank angle. Data are taken for different discharge valve seat designs, as shown by Figure 7. Each design is applied on the compressor, in order to know its effect on the compressor.

Given a SISO system as Figure 3, The output and the control input performances can be studied through 4 "sensitivity" functions only.

From Figure 3, it is obtained the output performance equations below,

$$
y(s)=\frac{1}{1+G(s) K(s)} G K_{r}+d_{y}-G K_{n}+G d_{i}
$$

or it can be represented as Figure 4 where $S$ is sensitivity function, $T$ is complementary sensitivity function, and $S(s)+T(s)=1$.

From Figure 4, the plant output $y(t)$ can track the reference $r(t)$ by making the complementary sensitivity function $T(s)$ equal to 1 . In the other side, the effect of the output disturbance $d_{y}(t)$ on the plant output $y(t)$ can be made small by making the sensitivity function $S(s)$ small, the same manner applies to $d_{i}(t)$. Lastly, the effect of the measurement noise $n(t)$ on the plant output $y(t)$ can be made small by making the complementary sensitivity function $T(s)$ small.

While, with the same manner, it is obtained the input performance equations as

$$
u(s)=\frac{1}{1+K(s) G(s)} K_{r}-K d_{y}-K_{n}-K G d_{i}
$$

or it can be represented as Figure 5 .

From Figure 5, The transfer function $K S(s)$ should be upper bounded so that $u(t)$ does not reach the physical constrains, even for a large reference $r(t)$.

In the other side, the effect of the input disturbance $d_{i}(t)$ on the plant input $u(t)$ can be made small by making the sensitivity function $S(s)$ small. Then, the effect of the measurement noise $n(t)$ on the plant input $u(t)$ can be made small by making the sensitivity function $K S(s)$ small (in high frequencies).

From the explanation above, it can be concluded that there is trade-off that can be reached with one aims:

a. to reject the disturbance effects in low frequency

b. to minimize the noise effects in high frequency We will require : 
a. S and SG to be small in low frequencies to reduce the load (output and input) disturbance effects on the controlled output

b. T and KS to be small in high frequencies to reduce the effects of measurement noises on the controlled output and on the control input (actuator efforts)

In terms of control synthesis, all these specifications can be tackled in the following problem: find $\mathrm{K}$ s.t

$$
\left\|\begin{array}{c}
W_{e} S \\
W_{u} K S \\
W_{T} T \\
W_{S G} S G
\end{array}\right\|_{\infty} \leq 1
$$

where, $\mathrm{W} *(\mathrm{~s})$ is a selected weighting function on each template on sensitivity function. However, the simpler following one is often studied such as, find K s.t

$$
\left\|\begin{array}{c}
W_{e} S \\
W_{u} K S
\end{array}\right\|_{\infty} \leq 1
$$

since the latter allows to consider the closed-loop output performance as well as the actuator constraints.

\section{Small Gain Theorem}

In order to assure that the system is stable, the small gain theorem approach is chosen. Consider the system shown by Figure 6 . Then, the small gain therorem is defined as,

c. Definition 3.

Small Gain Theorem: Suppose $M \in R H_{\infty}$. The closed-loop system is well-posed and internally stable for all $\Delta \in R H_{\infty}$ such that:

1. $\|\Delta\|_{\infty} \leq \delta$ if and only if $\|\mathrm{M}(\mathrm{s})\|_{\infty}<1 / \delta$

2. $\|\Delta\|_{\infty}<\delta$ if and only if $\|\mathrm{M}(\mathrm{s})\|_{\infty} \leq 1 / \delta$

The first step which is taken to analyse stability of the system is deciding the type of uncertainties. There are six types of uncertainty. In this report, only the output multiplicative uncertainty is presented. Figure 7 represents the block diagram of the system with uncertainty. With the output multiplicative uncertainty, it is assumed nominal stability is achieved, i.e $\mathrm{M} \in \mathrm{RH}_{\infty}$. Then, the closed-loop system is robustly stable, i.e internally stable for all $\Delta \mathrm{O} \in \mathrm{RH}_{\infty}$ such that $\left\|\omega_{\mathrm{O}} T_{y}\right\|_{\infty} \leq 1$ or it can be formed as :

$$
G_{p}=\left(I+\omega_{O} \Delta_{O}\right) G ; \quad \forall \Delta_{O} \text { s.t. }\left\|\Delta_{O}\right\|_{\infty} \leq 1 ; \quad C N S:\left\|\omega_{\mathrm{O}} T_{y}\right\|_{\infty} \leq 1
$$

where $G_{p}$ is system with all the possible uncertainties. $T_{y}$ is complementary sensitivity function and $\Delta$ is the parametric uncertainty.

\section{IMPLEMENTATION METHOD FOR THE SYSTEM}

The implementation method for the system is focused on the vehicle which run during acceleration. This chapter consists of two sections. The first section presents the residual synthesis for fault detection. The second section presents the $\mathrm{H}_{\infty}$ controller synthesis. Then, some analysis of the robust stability are presented in that section. Afterwards, the close loop response system will be presented.

\section{A. Detection of critical situations}

The system of longitudinal vehicle dynamics that depends on the scheduling parameter, $\rho(\mathrm{k})$ is given by

$$
\begin{gathered}
x(k+1)=A(\rho) x(k)+B(\rho) u(k)+E f(k) \\
y(k)=C x(k)+D u(k)+F f(k)
\end{gathered}
$$

Considering that the uncertainties change slowly in time, the observability matrix of the system is given by :

$$
O=\left[\begin{array}{c}
C \\
C A(\rho)
\end{array}\right]
$$

This system is observable with $\rho=\frac{1}{R \omega}$. Using only the wheel speed measurement, the matrix $\mathrm{H}_{\mathrm{os}}$ of the system with horizon $\mathrm{s}=3$ is :

$$
H_{o S}(\rho)=\left[\begin{array}{c}
C \\
C A(\rho) \\
C A^{2}(\rho)
\end{array}\right]
$$

Then, the matrix Hos is decomposed as :

$$
\begin{aligned}
& H_{O S_{1}}(\rho)=\left[\begin{array}{c}
C \\
C A(\rho)
\end{array}\right] \\
& H_{O S_{2}}(\rho)=\left\lfloor C A^{2}(\rho)\right\rfloor
\end{aligned}
$$

Then, the formula of the residual for this case is

$$
r(k)=W(\rho(k))^{T}\left(Y_{s}(k)-G_{A B D}(\rho(k)) U_{s}\right)
$$

In addition, it is necessary to determine the threshold $\left(r_{k}\right)$. The value of the threshold is chosen by the observation of the linear system in normal condition. Since, the condition of the system depends on the control input $\left(\mathrm{u}_{\mathrm{i}}\right)$, it is chosen 0.03 as an control input. Then, the fault detection approach shows that the residual remain small. It also indicates the limit of the residu is 0.023 . Therefore, it can be decided that the threshold $\left(r_{k}\right)$ is 0.023 .

In order to evaluate the performance of the detector of critical situation, 2 cases has been studied. The critical situation depends on the control input. One can clearly feel that abrupt wheel torque will cause critical situation. In this case, 2 situations are considered.

Firstly, a small input of $\mathrm{u}_{\mathrm{i}}=0.05$ is considered. The force is presented in the first figure in Figure 8. It can be shown that the force remains close to the linear one. The fault detection approach shows that the residual remain small.

Secondly, a more abrupt input, $\mathrm{u}_{\mathrm{i}}=0.1$ is considered. In this condition, the system run in normal area, critical area and skidding area as the left figure in Figure 9 shows. In this case, the system has some faults because of critical condition.

\section{B. $H_{\infty}$ Controller Synthesis}

In this section, it is presented the response of the closed loop system. There are two kinds of observation. Firstly, the observation about performance analysis using sensitivity function with the position of template is represented by Figure 10. This observation is applied to the linearized system with the operating point $\rho_{0}=0.27$. Therefore, the matrices of the linear system are presented as :

$$
\begin{aligned}
& A=\left[\begin{array}{cc}
-2.1475 & 0.0996 \\
100 & -7.5
\end{array}\right] \\
& B=\left[\begin{array}{c}
0 \\
644.084
\end{array}\right] \\
& C=\left[\begin{array}{ll}
0 & 1
\end{array}\right] \\
& D=0
\end{aligned}
$$


Secondly, the stability of the LPV system is proved thanks to the small gain theorem. In order to synthesis the controller, we use the theorem of performance analysis using sensitivity function. The weights are applied to the system as Figure 10 where The S, SG, T and KS curve are shown in Figure 11 with the configuration shown in Figure 10.

From the result, it is found that $\mathrm{T}$ is small in high frequencies. But $\mathrm{KS}$ is high in high frequencies. It means that the controller cannot reduce the effects of measurement noise on the controlled output and on the control input (actuator). In the other side, S and SG are small in low frequencies. It shows that the controller can reduce the load (output and input) disturbance effects on the controlled output. This result is not the best performance because it runs for a linear system. Considering that the plant is non linear, it is necessary to manipulate the bandwidth, in order to be able to cope the nonlinearity of the system.

In the section, the small gain theorem is used to analyse the robust stability. Due to the case that output multipicative uncertainty is applied to the system, then the nominal stability is reached when .

In order to apply the theorem, it is defined all the possibility of the scheduling parameter $(\rho)$ where $\rho=\rho 0$ $+\Delta . \rho$ is scheduling parameter where $\rho 0$ is nominal scheduling parameter and $\Delta$ is uncertainty. The first step, one must define boundary of rotational speed. For this vehicle, we define lower bound, $\omega=36.036 \mathrm{rad} / \mathrm{s}$ and upper bound $\omega=83.333 \mathrm{rad} / \mathrm{s}$. It is important to define the nominal rotational speed where. In this case, one choose $\omega 0=49.383$ so that $\rho 0=0.27$.

Finally, one check robust stability using small gain theorem. The aim of that is applying a given controller $\mathrm{K}$, we determine whether the system remains stable for all plants in the uncertainty set.

Using small gain theorem, one got the Figure $8 . G_{p}$ is all possibility of plant. From these figure, it is shown that condition nominal stability is reached because

$$
\frac{G(s)-G_{p}(s)}{G(s)}>\frac{1}{\Delta W 1}>T
$$

Afterward, the controller is simulated to the plant. The first experiment is to detect the normal and critical condition based on the input speed of the system. Based on the Figure 13, the greater the speed, the greater the faults even though the residual has relative small differences with the threshold.

Next, we observe the response of the system compared with applying other controller to verify the perfomance of the system in term of vehicle speed tracking. It is obtained the output of the system and residual of the system which are represented on Figure 14 and 15.

In this section, it is given the reference $10 \mathrm{~km} / \mathrm{h}$ for first 25 seconds, afterward, it is increased to be $20 \mathrm{~km} / \mathrm{h}$. From this figure, it is shown that there are faults when the reference changes. The first figure represents the closed-loop system with the LQR controller, and the second figure represents the closed-loop system with the $\mathrm{H} \infty$ robust controller. LQR controller cannot cope nonlinearity of the system, therefore, the faults occured after changing the reference. IAE (Integral Absolute Error) of this system is 28.25 . In the other hands, when the $\mathrm{H} \infty$ robust controller is applied to the system, the faults occured for 0.1 seconds even though it has high overshoot. And IAE of this system is 2.704. Thus, the implementation of the $\mathrm{H} \infty$ robust controller for longitudinal dynamics system has better result than implementation of LQR controller.

\section{CONCLUSION}

In this work, the problem of fault detection and accommodate the fault for LPV system have been solved. The longitudinal tire system is a non linear system which has two kinds of operation zone, normal and critical zone. The parity space approach detects the existence of faults on the critical zone. Therefore, the $\mathrm{H} \infty$ robust controller has been implemented to the plant in order to accomodate the faults.

The $\mathrm{H}_{\infty}$ robust controller is designed for linear system with manipulate the bandwidth $\omega_{\mathrm{b}}$ and $\omega_{\mathrm{bc}}$ in order to cope non linear problem. However, the result has been verified by small gain theorem and shows that the closed-loop system is nominal stable for all the possibility of schedulling parameter.

In order to test whether the performance of the closedloop system is good, the LQR controller is applied to the system. And the result shows that the response $\mathrm{H} \infty$ robust control is better than its LQR control system because $\mathrm{H} \infty$ robust controller can keep the system run on the normal zone.

\section{REFERENCES}

[1]. Varrier S., Koenig D., Martinez J Detection de Situation Critique d'Un Vehicule Automobile.

[2]. Rajamani R. (2012) "Vehicle Dynamics and Control, Mechanical Engineering Series". Springer.,

[3]. POUSSOT-VASSAL Charles. , (2008) Commande Robuste LPV Multivariable de Châssis Automobile.. These de Doctorat, Grenoble-INP.

[4]. Varrier S., Koenig D., Minh-Hieu Vu (2012). Detection de defauts sur systemes incertains par approche espace de parite. Manuscrit auteur, publié dans "Septième Conférence Internationale Francophone d'Automatique (CIFA 2012), Grenoble : France

[5]. Hagenblad A., Gustafsson F., Klein I. A Comparison of Two Methods for Stochastic Fault Detection: The Parity Space Approach and Principal Components Analysis. Department of Electrical Engineering, Link“ opings universitet, SE-581 83 Link oping, Sweden

[6]. Tan Kay Chen., Khor Eik Fun., Lee Tong Heng (2005) Multiobjective Evolutionary: Algorithms and Applications Springer

[7]. SZABOLCSI, RÓBERT., SZEGEDI PÉTER (2002) Robustness stability and robust performance of the automatic flight control systems AARMS volume 1, issue 2 (2002) 253-269. Hungary

[8]. ADOUNI Amel., BEN HAMED Mouna., SBITA Lassaâd APPLICATION OF PARITY SPACE APPROACH IN FAULT DETECTION OF DC MOTORS (2012) First International Conference on Renewable Energies and Vehicular Technology. 


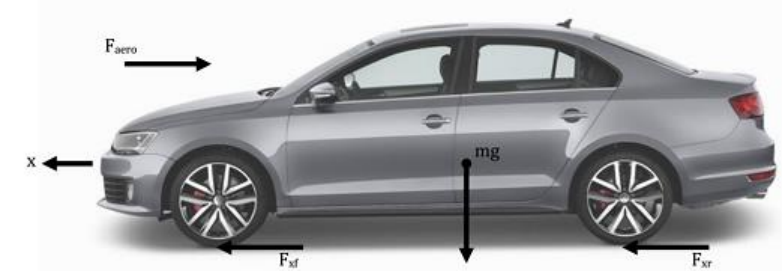

Figure 1. Longitudinal forces acting on a vehicle moving on an inclined road

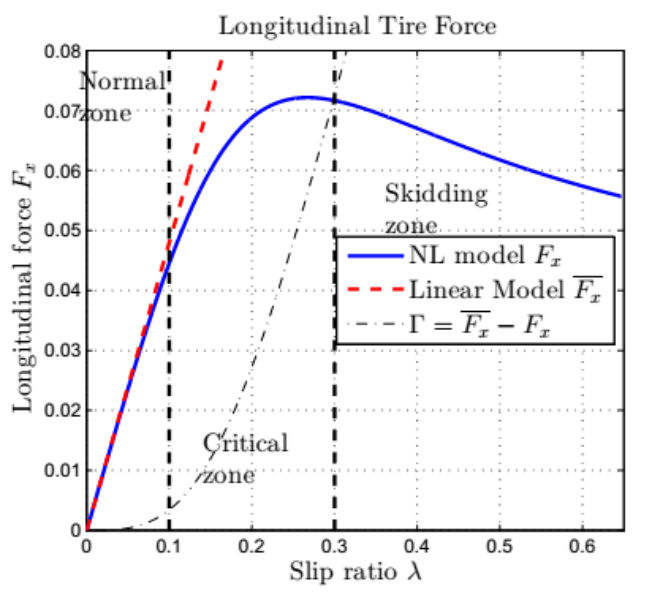

Figure 2. Longitudinal tire force

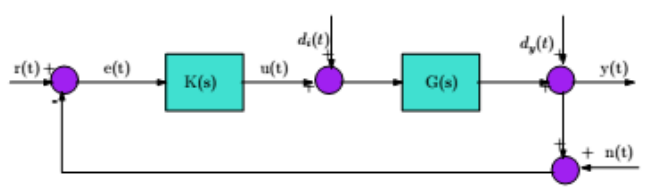

Figure 3. Control System Configuration

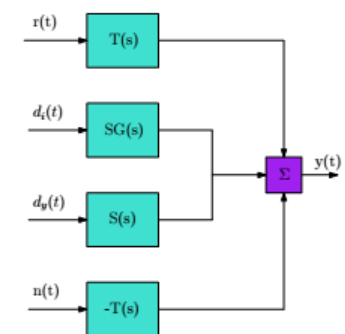

Figure 4. Output Performance

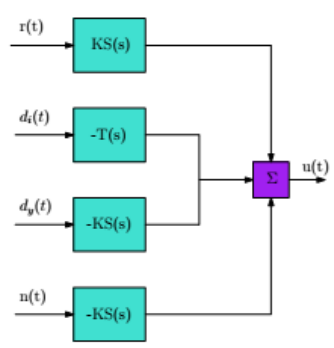

Figure 5. Input Performance

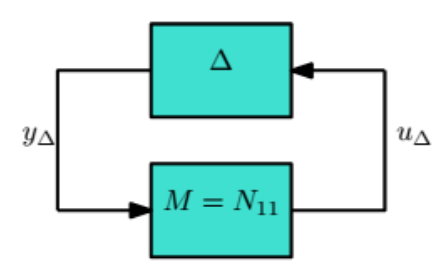

Figure 6. System with uncertainty
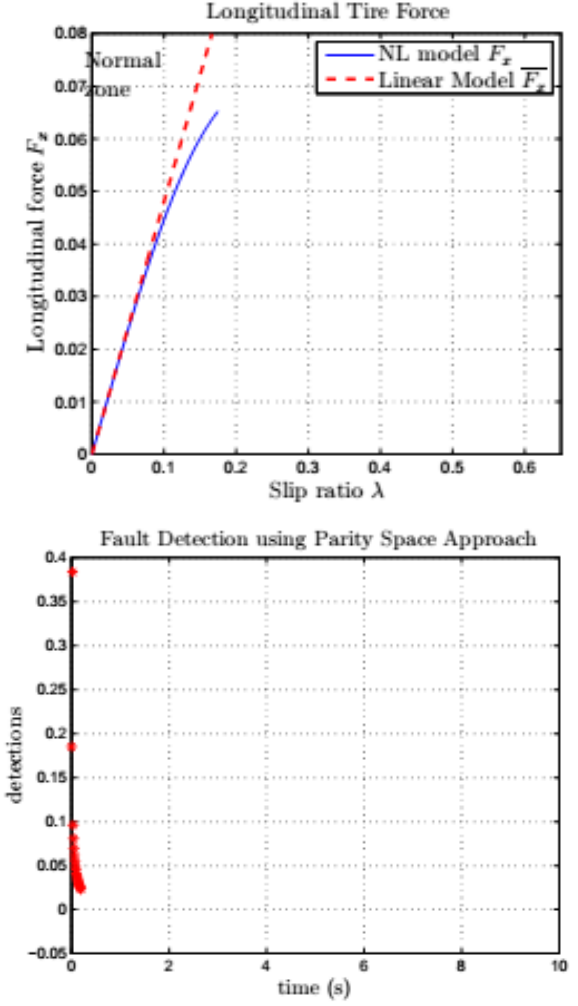

Figure 8. Condition 1 of The System
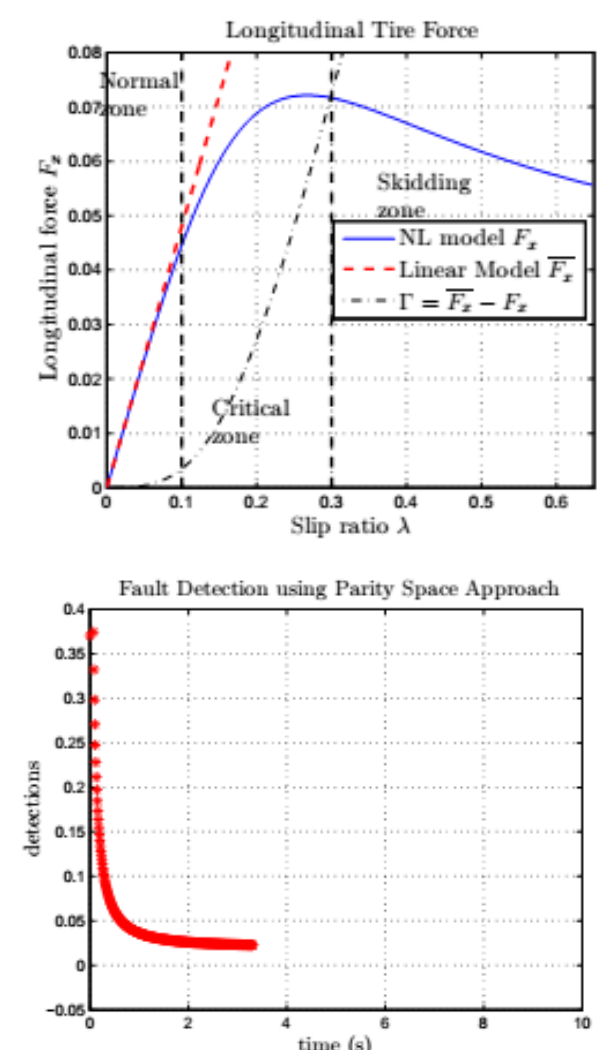

Figure 9. Condition 2 of The System

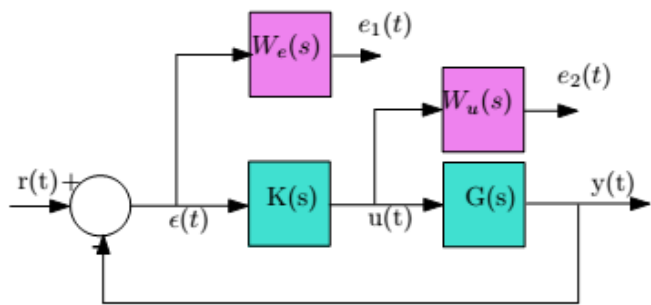

Figure 10 . The system with some weigths 

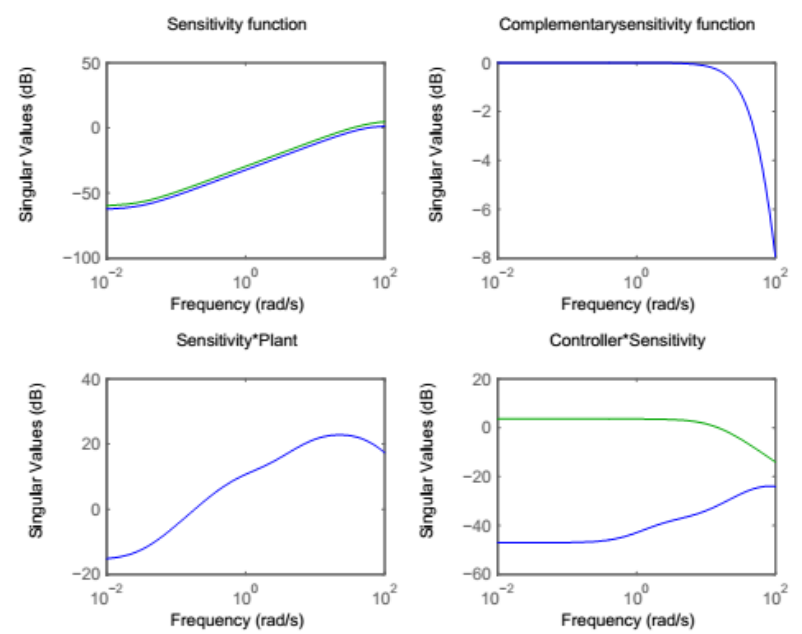

Figure 11. Performance Analysis using Sensitivity Function

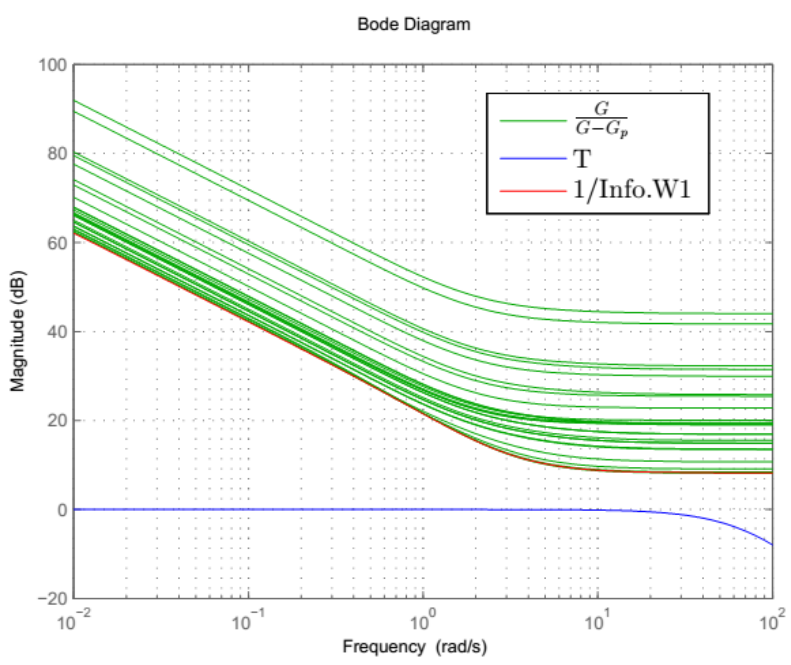

Figure 12. Analysis Robust Stability using Small Gain Theorem

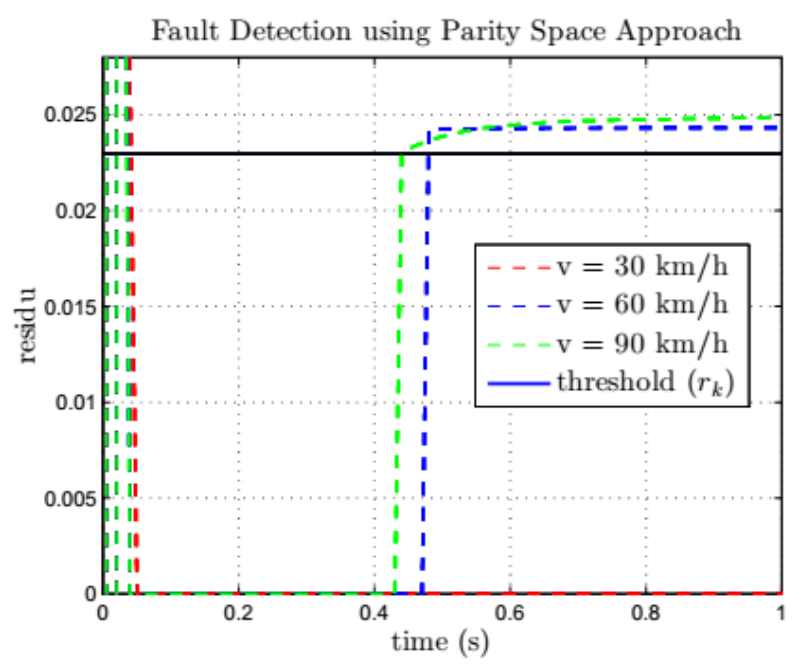

Figure 13. Analysis of Critical Situation

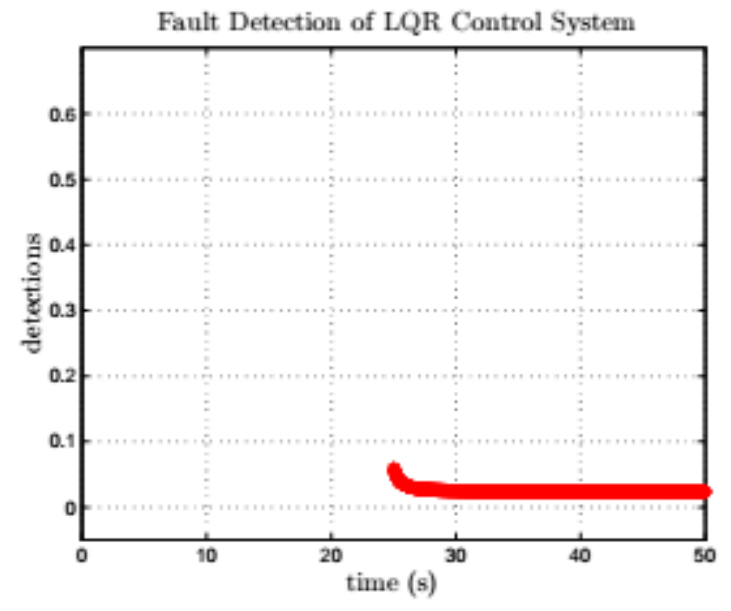

Figure 14. Output of the control system (1)

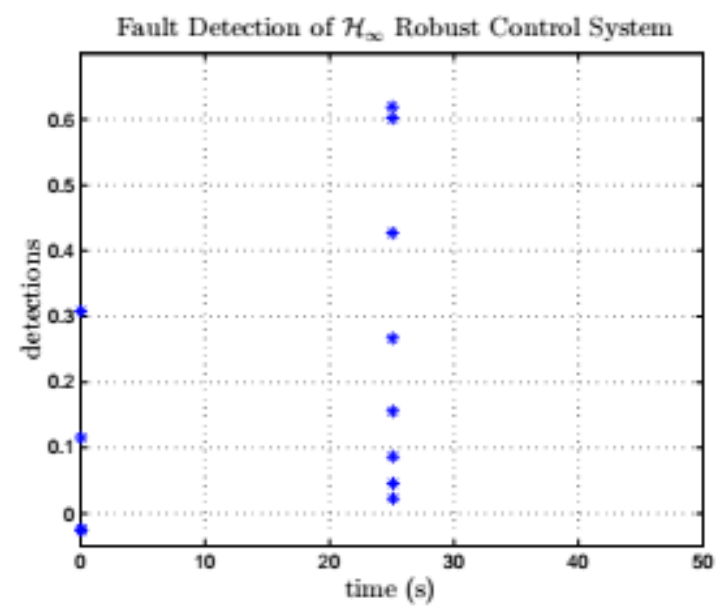

Figure 15. Output of the control system (2) 